\title{
On Conservation Forms and Invariant Solutions for Classical Mechanics Problems of Liénard Type
}

\author{
Gülden Gün Polat ${ }^{1}$ and Teoman Özer ${ }^{2}$ \\ ${ }^{1}$ Department of Mathematics, Faculty of Science and Letters, Istanbul Technical University, Maslak, 34469 Istanbul, Turkey \\ ${ }^{2}$ Division of Mechanics, Faculty of Civil Engineering, Istanbul Technical University, Maslak, 34469 Istanbul, Turkey \\ Correspondence should be addressed to Teoman Özer; tozer@itu.edu.tr
}

Received 27 March 2014; Accepted 12 June 2014; Published 7 August 2014

Academic Editor: Jian-Qiang Sun

Copyright (C) 2014 G. Gün Polat and T. Özer. This is an open access article distributed under the Creative Commons Attribution License, which permits unrestricted use, distribution, and reproduction in any medium, provided the original work is properly cited.

In this study we apply partial Noether and $\lambda$-symmetry approaches to a second-order nonlinear autonomous equation of the form $y^{\prime \prime}+f(y) y^{\prime}+g(y)=0$, called Liénard equation corresponding to some important problems in classical mechanics field with respect to $f(y)$ and $g(y)$ functions. As a first approach we utilize partial Lagrangians and partial Noether operators to obtain conserved forms of Liénard equation. Then, as a second approach, based on the $\lambda$-symmetry method, we analyze $\lambda$-symmetries for the case that $\lambda$-function is in the form of $\lambda\left(x, y, y^{\prime}\right)=\lambda_{1}(x, y) y^{\prime}+\lambda_{2}(x, y)$. Finally, a classification problem for the conservation forms and invariant solutions are considered.

\section{Introduction}

In classical mechanics, it is known that many important problems can be derived from Liénard equation of the form $y^{\prime \prime}+f(y) y^{\prime}+g(y)=0$. For instance, in dynamics, the Van der Pol oscillator that is a nonconservative oscillator with nonlinear damping is a physically important example of Liénard equation. Liénard equation can also be considered as a model for a spring-mass system where the damping force $f(y)$ corresponds to the position (e.g., the mass might be moving through a viscous medium of varying density), and the spring constant $g(y)$ corresponds to how much the spring is stretched. It is possible to present other similar examples related to the Liénard equation. Thus, it can be said that Liénard equation has an important role in mathematical physics and mechanics fields.

In addition, with respect to the investigation of solution of differential equations, one of the most powerful methods for nonlinear differential equations is based on the study of Lie group of transformations. In the last century, the applications of Lie groups to the problems in mechanics, mathematics, physics, and so forth, have been carried out by many mathematicians, for example [1-12]. For the case of Liénard equation, the classical Lie point symmetries of
Liénard equation are investigated in detail in the study [13]. However, the application of Noether theorem in the concept of theory of Lie groups to differential equations introduces that any Noether symmetry of the action of a physical system has a corresponding conservation law [1]. A conservation law means a quantity associated with a physical system that remains unchanged as the system evolves in time. In classical mechanics, the natural form of the Lagrangian is defined as the difference between the kinetic energy and potential energy of the system. An important property of the Lagrangian is that conservation laws can be easily derived from it. Furthermore, Noether theorem presents that variational symmetries are in one to one correspondence with conservation laws for the associated Euler-Lagrange equations. If the Lagrangian of a system is known, then the equations of motion of the system may be obtained by a direct substitution of the expression for the Lagrangian into the Euler-Lagrange equation. In addition, a Lagrangian of a system can be determined by making use of partial Lagrangian approach and the conservation laws can be obtained directly. The first study on this approach is carried out in [14]. In this study the Noether symmetries of Liénard equation are investigated for two different cases of arbitrary functions $f(y)$ and $g(y)$. In fact, $f(y)$ function 
is considered in two different forms for corresponding to two different mechanical problems, namely, linear undamped systems and linear damped systems. For both cases, the function $g(y)=k_{1} y+k_{2}$ can be chosen in a linear form for constant values of $k_{1}$ and $k_{2}$. The first case for the linear undamped systems corresponds to the equation of motion of the free particle and simple harmonic oscillator, and the second case for the linear damped systems corresponds to the damped harmonic oscillator. As a result, for different choices of $k_{1}$ and $k_{2}$, the conserved forms, partial Noether symmetries can be analyzed and classified for the Liénard equation.

In addition, in the literature, there is another approach called $\lambda$-symmetry, which is introduced by Muriel and Romero [15-18]. They introduce a new prolongation formula to investigate $\lambda$-symmetries for second order differential equations and they prove that although the equation has no Lie symmetries and by using the prolongation formula, then $\lambda$-symmetry of differential equation can be obtained and so the order of equation can be reduced. For convenience they consider a specific form (linear) for $\lambda$-function such as $\lambda\left(x, y, y^{\prime}\right)=\lambda_{1}(x, y) y^{\prime}+\lambda_{2}(x, y)$ [17]. For example, for the specific choices functions $f(y)$ and $g(y)$, the Liénard equations correspond to the general modified Emden-type equation and then its groups properties can be analyzed with linear term and constant external forcing via $\lambda$-symmetries. Moreover, it is possible to apply $\lambda$-symmetry approach to the Liénard equation by choosing other different forms of $f(y)$ function and one can examine different $g(y)$ functions corresponding to each $f(y)$ function. So we can obtain different types of Liénard equations having physical meaning and highly nonlinear forms and by $\lambda$ symmetry concept new solutions, conservation laws and classification properties of the Liénard equation can be investigated.

The outline of paper is as follows. In Section 2 we introduce some preliminaries about partial Noether theorem. Section 3 is devoted to determine conservation laws and partial Noether symmetries of some important problems in classical mechanics derived from Liénard equation. In Section 4 we present some fundamental definitions about $\lambda$-symmetry approach and the calculation procedure of the integration factor and the conservation laws from $\lambda$ symmetries algorithmically. In Section 5 we investigate conservation laws, integration factors, and invariant solutions by using $\lambda$-symmetry concept, as an alternative approach, of some mechanics problems considered in Section 3. Some important results in the study are discussed in Section 6

\section{Fundamental Definitions about Noether Theorem}

Let $x=\left(x^{1}, \ldots, x^{n}\right)$ be the independent variable with coordinates $x^{i}$ and $y=\left(y^{1}, \ldots, y^{m}\right)$ the dependent variable with coordinates $y^{\alpha}$. The derivatives of $y$ with respect to $x$ are

$$
y_{i}^{\alpha}=D_{i}\left(y^{\alpha}\right), \quad y_{i j}^{\alpha}=D_{j} D_{i}\left(y^{\alpha}\right), \ldots
$$

where

$$
D_{i}=\frac{\partial}{\partial x^{i}}+y_{i}^{\alpha} \frac{\partial}{\partial y^{\alpha}}+y_{i j}^{\alpha} \frac{\partial}{\partial y_{j}^{\alpha}}, \quad i=1, \ldots, n
$$

is the operator of total differentiation. Additionally, if $\mathscr{A}$ is a vector space of all differential functions of all finite orders, that is called the universal space and then the operators given below can be defined in the space $\mathscr{A}$.

Definition 1. For each $\alpha$ the Euler-Lagrange operator is defined by

$$
\frac{\delta}{\delta y^{\alpha}}=\frac{\partial}{\partial y^{\alpha}}+\sum_{s \geq 1}(-1)^{s} D_{i_{1}} \cdots D_{i_{s}} \frac{\partial}{\partial y_{i_{1} \cdots i_{s}}^{\alpha}}, \quad \alpha=1, \ldots, m .
$$

Definition 2. Generalized operator can be formulated as

$$
X=\xi^{i} \frac{\partial}{\partial x^{i}}+\eta^{\alpha} \frac{\partial}{\partial y^{\alpha}}, \quad \xi^{i}, \eta^{\alpha} \in \mathscr{A}
$$

and for convenience this operator can be written as

$$
X=\xi^{i} \frac{\partial}{\partial x^{i}}+\eta^{\alpha} \frac{\partial}{\partial y^{\alpha}}+\sum_{s \geq 1} \zeta_{i_{1} \cdots i_{s}}^{\alpha} \frac{\partial}{\partial y_{i_{1} \cdots i_{s}}^{\alpha}},
$$

where the additional coefficients are examined by the formula

$$
\begin{aligned}
\zeta_{i}^{\alpha} & =D_{i}\left(W^{\alpha}\right)+\xi^{j} y_{i j}^{\alpha}, \\
\zeta_{i_{1} \cdots i_{s}}^{\alpha} & =D_{i}\left(W^{\alpha}\right)+\xi^{j} y_{i j}^{\alpha}, \quad s \geq 2,
\end{aligned}
$$

in which $W^{\alpha}$ is the Lie characteristic function defined as

$$
W^{\alpha}=\eta^{\alpha}-\xi^{j} y_{j}^{\alpha}
$$

The generalized operator (5) can also be rewritten by using Lie characteristic function such as

$$
X=\xi^{i} D_{i}+W^{\alpha} \frac{\partial}{\partial y^{\alpha}}+\sum_{s \geq 1} D_{i_{1}} \cdots D_{i_{s}}\left(W^{\alpha}\right) \frac{\partial}{\partial y_{i_{1} \cdots i_{s}}^{\alpha}},
$$

and the Noether operator associated with a generalized operator $X$ is given by

$$
\begin{array}{r}
N=\xi^{i}+W^{\alpha} \frac{\partial}{\partial y^{\alpha}}+\sum_{s \geq 1} D_{i_{1}} \cdots D_{i_{s}}\left(W^{\alpha}\right) \frac{\delta}{\delta y_{i i_{1} \cdots i_{s}}^{\alpha}}, \\
i=1, \ldots, n,
\end{array}
$$

where the Euler-Lagrange operators with respect to derivatives of $y^{\alpha}$ are obtained from (3) by replacing $y^{\alpha}$ by the corresponding derivatives

$$
\begin{array}{r}
\frac{\delta}{\delta y_{i}^{\alpha}}=\frac{\partial}{\partial y^{\alpha}}+\sum_{s \geq 1}(-1)^{s} D_{j_{1}} \cdots D_{j_{s}} \frac{\partial}{\partial y_{i j_{1} \cdots j_{s}}^{\alpha}}, \\
i=1, \ldots, n, \alpha=1, \ldots, m .
\end{array}
$$


Definition 3. Suppose that a $n$ th-order ordinary differential equation system is given by

$$
f_{\alpha}\left(x, y, y_{(1)}, y_{(2)}, \ldots, y_{(n)}\right)=0, \quad \alpha=1,2, \ldots, m,
$$

with maximal rank and is locally solvable.

Definition 4. $I^{j}=I^{j}\left(x, y, y_{(1)}, y_{(2)}, \ldots, y_{(k-1)}\right) \in \mathscr{A}$ which satisfies what is given below

$$
D_{i}\left(I^{i}\right)=0
$$

is called a conservation law of (11). Moreover, (12) exists for all solutions of (11). In addition, (11) are assumed to be as the following form:

$$
f_{\alpha} \equiv f_{\alpha}^{0}+f_{\alpha}^{1}=0, \quad \alpha=1,2, \ldots, m .
$$

Let us consider differential equations of the form

$$
\frac{\delta L}{\delta y^{\alpha}}=0, \quad \alpha=1,2, \ldots, m,
$$

where $L=\left(L x,\left(x, y, y_{(1)}, \ldots, u_{(l)}\right)\right) \in \mathscr{A}, l \leq k, k$ being the order of (14), is a Lagrangian and $\delta / \delta y^{\alpha}$ is the Euler-Lagrange operator defined by (3).

Suppose that if nonzero functions $g_{\alpha}^{\beta} \in \mathscr{A}$ are defined such that (14)

$$
\frac{\delta L}{\delta y^{\alpha}}=g_{\alpha}^{\beta} f_{\beta}^{1}
$$

in which $f_{\beta}^{1} \neq 0$ then $L$ is called a partial Lagrangian of (13).

Definition 5. If there exists a vector $B=\left(B^{i}, \ldots, B^{n}\right), B^{i} \in \mathscr{A}$, $B^{i} \neq N^{i} L+C^{i}, C^{i}$ are constants and $W=\left(W^{1}, \ldots, W^{m}\right)$. If $W^{\alpha} \in \mathscr{A}$ is the characteristic of $X$ then

$$
X(L)+L D_{i}\left(\xi^{i}\right)=W^{\alpha} \frac{\delta L}{\delta y^{\alpha}}+D_{i}\left(B^{i}\right)
$$

is called Noether-type symmetry operator corresponding to a partial Lagrangian $L \in \mathscr{A}$. In addition, $B$ is called gauge function. If $X$ is a partial Noether operator corresponding to partial Lagrangian $L$ then the gauge function $B$ exists then the first integral is given by

$$
I=\xi L+\left(\eta-y^{\prime} \xi\right) L_{y^{\prime}}-B .
$$

\section{Partial Noether Symmetries of Liénard Equation}

Let us consider the one-dimensional nonlinear Liénard-type differential equation such as

$$
y^{\prime \prime}+f(y) y^{\prime}+g(y)=0
$$

where $f(y)$ and $g(y)$ are smooth functions of $y$. In this section we analyze the partial Noether symmetries of (18).
For the Liénard equation (18), the Euler-Lagrange operator can be written as (3)

$$
\frac{\delta}{\alpha y^{\alpha}}=\frac{\partial}{\partial y^{\alpha}}-D_{x} \frac{\partial}{\partial y_{x}}+D_{x}^{2} \frac{\partial}{\partial y_{x x}},
$$

and the partial Lagrangian $L$ for the Liénard equation (18) is given by

$$
L=\frac{1}{2} y^{\prime 2}-\int g(y) d y .
$$

Therefore (18) becomes

$$
\frac{\delta L}{\delta y}=-g(y)-y^{\prime}=y^{\prime} f(y)
$$

The partial Noether operators corresponding to $L$ are

$$
X(L)=\xi \frac{\partial}{\partial x}+\eta \frac{\partial}{\partial y}+\eta^{1} \frac{\partial}{\partial y^{\prime}},
$$

where $\eta^{1}$ is equal to

$$
\eta^{1}=\eta_{x}+\left(\eta_{y}-\xi_{x}\right) y^{\prime}-\xi_{y} y^{\prime 2}
$$

By using the relation (16) we have

$$
\begin{aligned}
\eta_{x} y^{\prime} & +\left(\eta_{y}-\xi_{x}\right) y^{\prime 2}-\xi_{y} y^{\prime 3}-\eta g(y) \\
& +\frac{1}{2} y^{\prime 3} \eta_{y}-\xi_{x} \int g(y) d y-y^{\prime} \xi_{y} \int g(y) d y \\
= & \eta f(y) y^{\prime}-\xi f(y) y^{\prime 2}+B_{x}+y^{\prime} B_{y}
\end{aligned}
$$

and analyzing (24) the coefficients of derivatives $y^{\prime}$ gives the following determining equations:

$$
\begin{gathered}
\xi_{y}=0, \\
\eta_{y}-\frac{1}{2} \xi_{x}+\xi f(y)=0, \\
\eta_{x}-\eta f(y)-B_{y}=0, \\
-\eta g(y)-\xi_{x} \int g(y) d y-B_{x}=0 .
\end{gathered}
$$

First, (25) gives

$$
\xi=a(x)
$$

where $a(x)$ is an arbitrary function. Then substituting $\xi$ into (26) $\eta$ gives

$$
\eta=\frac{1}{2} a(x)^{\prime} y-a(x) \int f(y) d y+\beta(x),
$$


where $\beta(x)$ is an arbitrary function. Differentiating (27) and (28), respectively, with respect to $x$ and $y$ we have the following equation:

$$
\begin{aligned}
g^{\prime}(y) & \left(a^{\prime}(x) y-2 a(x) \int f(y) d y+2 \beta(x)\right) \\
+ & g(y)\left(3 a^{\prime}(x)-2 a(x) f(y)\right) \\
+ & a^{\prime \prime \prime}(x) y-2 a^{\prime \prime}(x) \int f(y) d y+2 \beta^{\prime \prime}(x) \\
& -a^{\prime \prime}(x) y f(y)+2 a^{\prime}(x) f(y) \int f(y) d y \\
& -2 \beta^{\prime}(x) f(y)=0,
\end{aligned}
$$

which is a differential equation in terms of the unknown functions $f(y)$ and $g(y)$. In the following subsections we consider some classical mechanics problems related with Liénard-type differential equation for different choices of $f(y)$ and $g(y)$ functions.

3.1. Linear Undamped Systems: $f(y)=0$. If we consider $f(y)=$ 0 , then (31) is equal to

$$
\begin{gathered}
g^{\prime}(y)\left(2 \beta(x)+a^{\prime}(x) y\right)+3 a^{\prime}(x) g(y) \\
+a^{\prime \prime \prime}(x) y+2 \beta^{\prime \prime}(x)=0 .
\end{gathered}
$$

It is easy to see that a solution of (32) is

$$
g(y)=k_{1} y+k_{2}
$$

where $k_{1}$ and $k_{2}$ are arbitrary constants. We now analyze the following subcases:
(i) $k_{1}=k_{2}=0$,
(ii) $k_{1}=0$ and $k_{2} \neq 0$,
(iii) $k_{1} \neq 0$ and $k_{2}=0$,
(iv) $k_{1} \neq 0$ and $k_{2} \neq 0$.

Case 1 (Free particle motion $\left(k_{1}=k_{2}=0\right)$ ). If we take $k_{1}=k_{2}=0$, then (33) is equal to zero and thus the Liénard equation (18) becomes

$$
y^{\prime \prime}=0 .
$$

This form of (18) represents the equation of motion of a free particle. By using (32), (29), and (30) we obtain the partial Noether infinitesimals

$$
\xi=\frac{c_{1} x^{2}}{2}+c_{2} x+c_{3}, \quad \eta=\frac{\left(c_{1} x+c_{2}\right) y}{2}+c_{4} x+c_{5},
$$

where $c_{1}, c_{2}, c_{3}, c_{4}$, and $c_{5}$ are arbitrary constants. The corresponding partial Noether symmetry generators are

$$
\begin{gathered}
X_{1}=\frac{x^{2}}{2} \frac{\partial}{\partial x}+\frac{x y}{2} \frac{\partial}{\partial y}, \quad X_{2}=x \frac{\partial}{\partial x}+\frac{y}{2} \frac{\partial}{\partial y}, \\
X_{3}=\frac{\partial}{\partial x}, \quad X_{4}=x \frac{\partial}{\partial y}, \quad X_{5}=\frac{\partial}{\partial y} .
\end{gathered}
$$

Additionally, the gauge function is obtained from (25) and (26)

$$
B(x, y)=\frac{1}{4} c_{1} y^{2}+c_{4} y .
$$

The corresponding conservation laws by the (17) formula are obtained in the following forms:

$$
\begin{gathered}
I_{1}=-\frac{y^{2}}{4}+\frac{1}{2} x y y^{\prime}-\frac{1}{4} x^{2}\left(y^{\prime}\right)^{2}, \\
I_{2}=\frac{y y^{\prime}}{2}-\frac{1}{2} x\left(y^{\prime}\right)^{2}, \quad I_{3}=-\frac{1}{2} x\left(y^{\prime}\right)^{2}, \\
I_{4}=-y+x y^{\prime}, \quad I_{5}=y^{\prime} .
\end{gathered}
$$

It is important to mention that each conservation law satisfies the equality $D_{x} I=W\left(-y^{\prime \prime}\right)=0$ with respect to the characteristic $W$.

Case 2 (Free falling particle $\left(k_{1}=0, k_{2} \neq 0\right)$ ). For this case of $k_{1}$ and $k_{2}(18)$ becomes

$$
y^{\prime \prime}+k_{2}=0
$$

which corresponds to the equation of motion of a free falling particle. By solving (25)-(28) we get the $\xi, \eta$ and gauge function $B(x, y)$

$$
\begin{gathered}
\xi=\frac{c_{1} x^{2}}{2}+c_{2} x+c_{3}, \\
\eta=\frac{\left(c_{1} x+c_{2}\right) y}{2}+c_{3} x+c_{4}-\frac{3}{4} c_{2} x^{2} k_{2}-\frac{1}{4} c_{1} x^{3} k_{2} \\
B=\frac{1}{16}\left(16 c_{5}+16 c_{3} y+4 c_{1} y^{2}-16 c_{4} x k_{2}\right. \\
-8 c_{3} x^{2} k_{2}-24 c_{2} x y k_{2} \\
\left.-12 c_{1} x^{2} y k_{2}+4 c_{2} x^{3} k_{2}^{2}+c_{1} x^{4} k_{2}^{2}\right) .
\end{gathered}
$$

The corresponding partial Noether symmetry generators are

$$
\begin{aligned}
& X_{1}=\frac{x^{2}}{2} \frac{\partial}{\partial x}+\left(\frac{x y}{2}-\frac{x^{3} k_{2}}{4}\right) \frac{\partial}{\partial y}, \\
& X_{2}=x \frac{\partial}{\partial x}+\left(\frac{y}{2}-\frac{3 x^{2} k_{2}}{4}\right) \frac{\partial}{\partial y} \\
& X_{3}=\frac{\partial}{\partial x}+y \frac{\partial}{\partial y}, \quad X_{4}=\frac{\partial}{\partial y}
\end{aligned}
$$

and the conservation laws corresponding to each partial Noether symmetries are

$$
\begin{aligned}
I_{1}=\frac{1}{16}( & -x^{4} k_{2}^{2}-4 y^{2}-4 x^{3} k_{2} y^{\prime} \\
& \left.-4 x^{2} k_{2} y^{\prime 2}+4 x y\left(x k_{2}+2 y^{\prime}\right)\right),
\end{aligned}
$$




$$
\begin{aligned}
& I_{2}=\frac{1}{4}\left(-x^{3} k_{2}^{2}-3 x^{2} k_{2} y^{\prime}-2 x y^{\prime 2}+2 y\left(x \lambda_{2}+y^{\prime}\right)\right), \\
& I_{3}=\frac{1}{2}\left(x^{2} k_{2}-2\left(1+k_{2}\right) y+2 x y^{\prime}-\left(y^{\prime}\right)^{2}\right), \\
& I_{4}=y^{\prime}+x k_{2} .
\end{aligned}
$$

Case 3 (Free linear harmonic oscillator $\left(k_{1} \neq 0, k_{2}=0\right)$ ). If we chose $k_{1}$ as an arbitrary and $k_{2}=0$ thus the Liénard equation (18) is equal to

$$
y^{\prime \prime}+k_{1} y=0
$$

which refers to the equation of a linear harmonic oscillator for $k_{1}>0$. Then the solution of determining (25)-(28) for the $f(y)=0$ and $g(y)=k_{1} y$ gives

$$
\begin{gathered}
\xi=c_{3}-\frac{c_{2} \cos \left(2 x \sqrt{k_{1}}\right)}{2 \sqrt{k_{1}}}+\frac{c_{1} \sin \left(2 x \sqrt{k_{1}}\right)}{2 \sqrt{k_{1}}}, \\
\eta=c_{4} \cos \left(x \sqrt{k_{1}}\right)+c_{5} \sin \left(x \sqrt{k_{1}}\right) \\
+\frac{1}{2} y\left(c_{1} \cos \left(2 x \sqrt{k_{1}}\right)+c_{1} \cos \left(2 x \sqrt{k_{1}}\right)\right), \\
B=\frac{1}{2 \sqrt{k_{1}}}\left(2 c_{6} \sqrt{k_{1}}+2 c_{5} k_{1} \cos \left(x \sqrt{k_{1}}\right)\right. \\
\quad+c_{2}\left(k+y^{2} k_{1}\right) \cos \left(2 x \sqrt{k_{1}}\right) \\
\quad-2 c_{4} y k_{1} \sin \left(x \sqrt{k_{1}}\right) \\
\left.\quad-k c_{1} \sin \left(2 x \sqrt{k_{1}}\right)-c_{1} y^{2} k_{1} \sin \left(2 x \sqrt{k_{1}}\right)\right) .
\end{gathered}
$$

The corresponding partial Noether symmetry generators are found as follows:

$$
\begin{gathered}
X_{1}=\frac{\sin \left(2 x \sqrt{k_{1}}\right)}{2 \sqrt{k_{1}}} \frac{\partial}{\partial x}+\frac{\cos \left(2 x \sqrt{k_{1}}\right)}{2} y \frac{\partial}{\partial y} \\
X_{2}=-\frac{\cos \left(2 x \sqrt{k_{1}}\right)}{2 \sqrt{k_{1}}} \frac{\partial}{\partial x}+\frac{\sin \left(2 x \sqrt{k_{1}}\right)}{2} y \frac{\partial}{\partial y}, \\
X_{3}=\frac{\partial}{\partial x}, \quad X_{4}=\cos \left(x \sqrt{k_{1}}\right) \frac{\partial}{\partial y} \\
X_{5}=\sin \left(x \sqrt{k_{1}}\right) \frac{\partial}{\partial y}
\end{gathered}
$$

and the conservation laws are

$$
\begin{aligned}
I_{1}=\frac{1}{4} & \left(\sqrt{k_{1}} \sin \left(2 x \sqrt{k_{1}}\right) y^{2}\right. \\
& \left.+2 y y^{\prime} \cos \left(2 x \sqrt{k_{1}}\right)-\frac{\sin \left(2 x \sqrt{k_{1}}\right) y^{2}}{\sqrt{k_{1}}}\right),
\end{aligned}
$$

$$
\begin{aligned}
& I_{2}=\frac{1}{4}(-\sqrt{k_{1}} \cos \left(2 x \sqrt{k_{1}}\right) y^{2} \\
&\left.+2 y y^{\prime} \sin \left(2 x \sqrt{k_{1}}\right)+\frac{\cos \left(2 x \sqrt{k_{1}}\right) y^{2}}{\sqrt{k_{1}}}\right), \\
& I_{3}=-\frac{1}{2}\left(-2 k+y^{2} k_{1}-\left(y^{\prime}\right)^{2}\right) \\
& I_{4}=\sqrt{k_{1}} \sin \left(x \sqrt{k_{1}}\right) y+\cos \left(x \sqrt{k_{1}}\right) y^{\prime}, \\
& I_{5}=-\sqrt{k_{1}} \cos \left(x \sqrt{k_{1}}\right) y+\sin \left(x \sqrt{k_{1}}\right) y^{\prime},
\end{aligned}
$$

in which it is easy to see that the relation $D_{x} I=0$ is satisfied for each conserved form.

Case 4 (Displaced linear harmonic oscillator $\left(k_{1} \neq k_{2} \neq 0\right)$ ). If the $k_{1}$ and $k_{2}$ are assumed to be arbitrary, then we obtain the Liénard equation (18) as the following form:

$$
y^{\prime \prime}+k_{1} y+k_{2}=0
$$

which represents a displaced simple harmonic motion. Equation (47) transforms the previous case with a change of variable such as $y \rightarrow \tilde{y}=y+\left(k_{2} / k_{1}\right)$. Therefore, this form of Liénard equation gives the similar partial Noether symmetries, generators, and conservation laws to Case 3.

3.2. Linear Damped Systems: $f(y)=$ Constant $=k$. The other case for $f(y)$ is to be considered as a constant. By substituting $f(y)=k$ into the (29) and (30) then we calculate the infinitesimals

$$
\begin{gathered}
\xi=a(x), \\
\eta=\left(\frac{1}{2} a^{\prime}(x)-a(x) k\right) y+\beta(x),
\end{gathered}
$$

and rewrite (31), which includes the unknown functions $f(y)$ and $g(y)$

$$
\begin{aligned}
g^{\prime}(y) & \left(2 \beta(x)-2 k a(x) y+a^{\prime}(x) y\right) \\
& +g(y)\left(3 a^{\prime}(x)-2 k a(x)\right) \\
& +a^{\prime}(x) 2 k^{2} y-2 k \beta^{\prime}(x)-3 k y a^{\prime \prime}(x)+2 \beta^{\prime \prime}(x) \\
& +y a^{\prime \prime \prime}(x)=0 .
\end{aligned}
$$

If we analyze the solutions of (49) then a solution of this equation for $g(y)$ can be considered as linear function of $y$, which is similar to the section $A$; that is,

$$
g(y)=k_{1} y+k_{2}
$$

where $k_{1}$ and $k_{2}$ are arbitrary constants. To examine all possibilities of $g(y)$ we again consider the following subcases:

(i) $k_{1}=k_{2}=0$, 
(ii) $k_{1}=0$ and $k_{2} \neq 0$,

(iii) $k_{1} \neq 0$ and $k_{2}=0$,

(iv) $k_{1} \neq 0$ and $k_{2} \neq 0$.

Case 1 (Free particle in a viscous medium $\left(k_{1}=k_{2}=0\right)$ ). For this case the Liénard equation (18) is equal to

$$
y^{\prime \prime}+k y^{\prime}=0
$$

which represents the equation of a free particle in a viscous medium. If we substitute $g(y)=0$ into (49), then by using the determining (27)-(28) we find the partial Noether symmetries $\xi, \eta$ and gauge function $B(x, y)$ such that

$$
\begin{aligned}
\xi & =\frac{e^{2 k x}}{2 k} c_{1}+\frac{e^{2 x}}{k} c_{2}+c_{3}, \\
\eta & =-\frac{1}{2}\left(c_{2} e^{k x}+23 k\right) y+c_{4} \frac{e^{k x}}{k}+c_{5}, \\
B(x, y) & =-\frac{1}{2 k}\left(2 c_{2} e^{k x}+c_{1} e^{2 k x}+2 c_{5} k^{2} y-c_{3} k^{3} y^{2}\right),
\end{aligned}
$$

and partial Noether symmetry generators are

$$
\begin{gathered}
X_{1}=\frac{e^{2 k x}}{2 k} \frac{\partial}{\partial x}, \quad X_{2}=\frac{e^{k x}}{k} \frac{\partial}{\partial x}-\frac{e^{k x} y}{2} \frac{\partial}{\partial y}, \\
X_{3}=-k y \frac{\partial}{\partial y}, \quad X_{4}=\frac{e^{k x}}{k} \frac{\partial}{\partial y}, \quad X_{5}=\frac{\partial}{\partial y} .
\end{gathered}
$$

By applying the definition of first integral (17) we obtain the conservation laws

$$
\begin{gathered}
I_{1}=-\frac{1}{4 k} e^{2 k x} y^{\prime 2}, \quad I_{2}=-\frac{e^{k x} y^{\prime}}{2 k}\left(y+k y^{\prime}\right), \\
I_{3}=-\frac{1}{2}\left(2+k^{2} y^{2}+2 k y y^{\prime}-y^{\prime 2}\right), \\
I_{4}=\frac{1}{k} e^{k x} y^{\prime}, \quad I_{5}=y+k y^{\prime},
\end{gathered}
$$

which satisfy the relation $D_{x} I=0$.

Case 2 (Falling particle in a viscous medium $\left(k_{2} \neq 0, k_{1}=0\right)$ ). If the functions $f(y)=k$ and $g(y)=k_{2}$ are substituted into the Liénard equation (18) we obtain

$$
y^{\prime \prime}+k y^{\prime}+k_{2}=0
$$

which is the equation of a falling particle in a viscous medium. It is obvious that this form of Liénard equation (55) can be transformed into previous case by using the simple transformation such as $y \rightarrow \tilde{y}=y+\left(k_{2} / k\right) w$. Therefore, if this transformation can be applied for (52)-(54) then partial Noether symmetries, partial Noether generators, and conservation laws are derived, respectively, in terms of $w$ for (55).

Case 3 (Damped linear harmonic oscillator $\left(k_{1} \neq 0, k_{2}=0\right)$ ). Suppose that $k_{1}$ is arbitrary and $k_{2}$ is equal to zero and thus Liénard equation (18) can be obtained as the form

$$
y^{\prime \prime}+k y^{\prime}+k_{1} y=0
$$

which corresponds to the equation of a damped linear harmonic oscillator. By considering similar process to previous cases we get partial Noether symmetries, partial Noether generators, and gauge function

$$
\begin{gathered}
\xi=c_{1} e^{x(k-\gamma)}+c_{2} e^{x(k+\gamma)}+c_{3} e^{k x}, \\
\eta=-\frac{1}{2}\left(2 c_{4} e^{1 / 2 x(k-\gamma)}+2 c_{5} e^{1 / 2 x(k+\gamma)}-e^{x(k-\gamma)} y\right. \\
\times\left(e^{x \gamma}\left(c_{3} k-c_{2} e^{x \gamma}(\gamma-k)\right)\right. \\
\left.\left.+c_{1}(k+\gamma)\right)\right) \\
B(x, y) \quad \frac{1}{4} e^{-x \gamma}\left(-4 c e^{k x}\left(c_{1}+e^{x \gamma}\left(c_{3}+c_{2} e^{x \gamma}\right)\right)\right. \\
+y\left(2 c_{5} e^{1 / 2 x(k+3 \gamma)}\right. \\
\times\left(-k+\gamma-2 c_{4} e^{1 / 2 x(k+\gamma)}\right. \\
\times(k+\gamma)+e^{k x} y \\
\times\left(c_{1}\left(k^{2}+k \gamma-4 k_{1}\right)-c_{2} e^{2 x \gamma}\right. \\
\left.\left.\left.\left.\times\left(-k^{2}+k \gamma+4 k_{1}\right)\right)\right)\right)\right)
\end{gathered}
$$

where $c_{1}, c_{2}, c_{3}, c_{4}$, and $c_{5}$ are arbitrary constants and $\gamma=$ $\sqrt{k^{2}-4 k_{1}} ; k^{2}-4 k_{1}$ is assumed to be positive. And the partial Noether generators are

$$
\begin{gathered}
X_{1}=e^{x(k-\gamma)} \frac{\partial}{\partial x}-\frac{1}{2} e^{x(k-\gamma)} y(k+\gamma) \frac{\partial}{\partial y}, \\
X_{2}=e^{x(k+\gamma)} \frac{\partial}{\partial x}+\frac{1}{2} e^{x(k-\gamma)+2 x \gamma} y(\gamma-k) \frac{\partial}{\partial y}, \\
X_{3}=e^{k x} \frac{\partial}{\partial x}-\frac{1}{2} e^{k x} k y \frac{\partial}{\partial y}, \quad X_{4}=e^{1 / 2 x(k-\gamma)} \frac{\partial}{\partial y}, \\
X_{5}=e^{1 / 2 x(k 4 \gamma)} \frac{\partial}{\partial y},
\end{gathered}
$$

and the corresponding conservation laws are

$$
\begin{aligned}
& I_{1}=-\frac{1}{4} e^{x(k-\gamma)}\left(\left(k^{2}+k \gamma-2 k_{1}\right) y^{2}+2\left(k+\gamma y y^{\prime}+2 y^{\prime 2}\right)\right), \\
& I_{2}=\frac{1}{4} e^{x(k+\gamma)}\left(\left(-k^{2}+k \gamma+2 k_{1}\right) y^{2}+2(\gamma-k) y y^{\prime}-2 y^{\prime 2}\right), \\
& I_{3}=-\frac{1}{2} e^{k x}\left(k_{1} y^{2}+k y y^{\prime}+y^{\prime 2}\right) \\
& I_{4}=\frac{1}{2} e^{x(k-\gamma)}\left((k+\gamma) y+2 y^{\prime}\right) \\
& I_{5}=\frac{1}{2} e^{x(k+\gamma)}\left((k-\gamma) y+2 y^{\prime}\right) .
\end{aligned}
$$


Case 4 (Displaced damped harmonic oscillator $\left(k_{1} \neq k_{2} \neq\right.$ $0))$. For this case we get the Liénard equation (18)

$$
y^{\prime \prime}+k y^{\prime}+k_{1} y+k_{2}=0
$$

which is the equation of displaced damped harmonic oscillator. By using the change of variable such as $y \rightarrow \tilde{y}=y+$ $k_{2} / k_{1}$ this case corresponds to the previous form of Liénard equation. So by applying this transformation to (57)-(59) partial Noether operators, partial Noether generators, and conservation laws can be obtained similarly.

\section{4. $\lambda$-Symmetry Approach for Differential Equations}

In this section we consider $\lambda$-symmetry properties of Liénard equation and for this purpose we first present some fundamental definitions and theorems about $\lambda$-symmetries $[15,17]$. Let us consider a $n$ th-order ordinary differential equation

$$
\widetilde{\Delta}\left(x, y^{(n)}\right)=0
$$

where variables $(x, y)$ are in some open set $M \subset X \times Y \cong \mathbb{R}^{2}$. For $k \in \mathbb{N}, M^{(k)} \subset X \times Y^{(k)}$ denotes the corresponding $k$ jet space and the elements of $M^{(k)}$ are denoted by $\left(x, y^{(k)}\right)=$ $\left(x, y, y^{\prime}, \ldots, y^{k}\right)$. By applying implicit function theorem to (61), as a result, this equation can be written in the explicit form

$$
y^{(n)}=\phi\left(x, y^{(n-1)}\right)
$$

The vector field

$$
A=\frac{\partial}{\partial x}+y^{\prime} \frac{\partial}{\partial y}+\cdots+\phi\left(x, y^{(n-1)}\right) \frac{\partial}{\partial y^{(n-1)}}
$$

is called the vector field associated with (62). An integrating factor of (61) is assumed to be such as $\mu\left(x, y^{(k)}\right)$ for some $k$ and $0 \leq k \leq n$. If the left-hand side of (61) is multiplied by integration factor $\mu$, total derivative of some function $\Delta\left(x, y^{(n-1)}\right)$ is obtained as the form

$$
\mu\left(x, y^{(k)}\right) \cdot \tilde{\Delta}\left(x, y^{(n)}\right)=D_{x}\left(\Delta\left(x, y^{(n-1)}\right)\right) .
$$

It is clear that $\lambda$-symmetries can be used for reduction of order of a differential equation. Namely, $D_{x}\left(\Delta\left(x, y^{(n-1)}\right)\right)=0$ is an exact differential equation, which is the result of trivial reduction of order $\Delta\left(x, y^{(n-1)}\right)=C, C \in \mathbb{R}$ [18]. If $\lambda \in$ $C^{\infty}\left(M^{(k)}\right), 0 \leq k \leq n$, is any solution of the partial differential equation:

$$
\sum_{i=0}^{n-1}\left(D_{x}+\lambda\right)^{i}(1) \frac{\partial \Delta}{\partial y^{(i)}}=0
$$

when $D_{x}\left(\Delta\left(x, y^{(n-1)}\right)\right)=0$, then the vector field $v=\partial_{y}$ is a $\lambda$-symmetry of (61).

Theorem 6 (see [17]). Assume that (61) is a nth-order ordinary differential equation that admits an integrating factor $\mu$ such that $\left.\mu\right|_{\widetilde{\Delta}=0} \neq 0$. If $\lambda$ is any particular solution of (65), then the vector field $\nu=\partial_{y}$ is $\lambda$-symmetry of (61).
Now let us consider the second order differential equation (62) and a vector field (63)

$$
\begin{gathered}
y^{\prime \prime}=\phi\left(x, y, y^{\prime}\right) \\
A=\frac{\partial}{\partial x}+y^{\prime} \frac{\partial}{\partial y}+\cdots+\phi\left(x, y, y^{\prime}\right) \frac{\partial}{\partial y^{\prime}}
\end{gathered}
$$

then a conservation law (first integral) of (66) is any function such as $I\left(x, y, y^{\prime}\right)$ which satisfies the relation

$$
A(I)=0
$$

If $\nu$ is assumed to be a $\lambda$-symmetry of (66) and $\omega\left(x, y, y^{\prime}\right)$ is a first-order invariant of $\nu^{[\lambda,(1)]}$ and any particular solution of the equation

$$
\omega_{y}+\lambda\left(x, y, y^{\prime}\right) \omega_{y^{\prime}}=0
$$

then a first-order invariant reduced equation of the form $\Delta_{R}\left(x, y, y^{\prime}\right)=0$ can be obtained by using the reduction process associated with the $\lambda$-symmetry. Thus the general solution is found such as an equation of the implicit form

$$
G(x, \omega)=C, \quad C \in \mathbb{R} .
$$

It is clear that $D_{x}\left(G\left(x, \omega\left(x, y, y^{\prime}\right)\right)=0\right.$ is an equivalent form of (66). Consequently,

$$
\mu\left(x, y, y^{\prime}\right)=G_{\omega}\left(x, y, \omega\left(x, y, y^{\prime}\right)\right) \cdot \omega_{y^{\prime}}\left(x, y, y^{\prime}\right)
$$

is an integrating factor of (66).

Theorem 7 (see [17]). Let $y^{n}=\phi\left(x, y^{(n-1)}\right)$ be a nth-order ordinary differential equation, where $\phi$ is an analytic function of its arguments. There exists a function $\lambda\left(x, y^{(k)}\right)$, for some $k<n$, such that the vector field $\nu=\partial_{y}$ is a $\lambda$-symmetry of the equation.

\section{5. $\lambda$-Symmetries, Conservation Laws, and Integrating Factors of Liénard Equation}

In this section we investigate $\lambda$-symmetries of (18) for different cases of arbitrary functions of $f(y)$ and $g(y)$. By considering theorem (62) the vector field $v=\partial_{y}$ is assumed to be as a $\lambda$-symmetry of (18). If we write explicit form of (18) corresponding to (66) we obtain

$$
\phi\left(x, y, y^{\prime}\right)=-y^{\prime} f(y)-g(y) .
$$

By applying (65) to (72) we find

$$
\lambda_{x}+y^{\prime} \lambda_{y}+\phi \lambda_{y^{\prime}}+\lambda^{2}-\phi_{y}-\lambda \phi_{y^{\prime}}=0,
$$

in which $\lambda$ is any particular solution of (73). For convenience a solution of $\lambda$ of (73) can be assumed to be linear form such that

$$
\lambda\left(x, y, y^{\prime}\right)=\lambda_{1}(x, y) y^{\prime}+\lambda_{2}(x, y) .
$$


Therefore, the expansion of (73) becomes

$$
\begin{gathered}
-g(y) \lambda_{1}+y^{\prime 2} \lambda_{1}^{2}+f(y) \lambda_{2}+2 y^{\prime} \lambda_{1} \lambda_{2} \\
+\lambda_{2}^{2}+y^{\prime} f^{\prime}(y)+g^{\prime}(y)+y^{\prime 2} \lambda_{1 y} \\
+y^{\prime} \lambda_{2 y}+y^{\prime} \lambda_{1 x}+\lambda_{2 x}=0 .
\end{gathered}
$$

The usual separation of powers of derivatives of $y(75)$ reduces to the system

$$
\begin{gathered}
\lambda_{1}^{2}+\lambda_{1 y}=0 \\
2 \lambda_{1} \lambda_{2}+f^{\prime}(y)+\lambda_{2 y}+\lambda_{1 x}=0 \\
-g^{\prime}(y)-g(y) \lambda_{1}+f(y) \lambda_{2}+\lambda_{2}^{2}+\lambda_{2 x}=0 .
\end{gathered}
$$

A particular solution of (76) is found

$$
\lambda_{1}(x, y)=\frac{1}{y} \text {. }
$$

And so (77) becomes

$$
\frac{2 \lambda_{2}}{y}+f^{\prime}(y)+\lambda_{2 y}=0
$$

To obtain the solution of (80) different cases of $f(y)$ should be considered. Firstly in order to compare Noether and $\lambda$ symmetry approaches we investigate the similar cases of functions $f(y)$ and $g(y)$, which are analyzed in Section 3.

Case 1. In this case we analyze $f(y)$ and $g(y)$ functions which correspond to the case of Section 3.1. Namely, $f(y)=0$ and $g(y)=k_{1} y+k_{2}$, which represent linear undamped systems. For this functions the determining equations (80) and (78) become

$$
\begin{gathered}
\frac{2 \lambda_{2}}{y}+\lambda_{2 y}=0 \\
-k_{1}-\left(k_{1} y+k_{2}\right) \lambda_{1}+\lambda_{2}^{2}+\lambda_{2 x}=0 .
\end{gathered}
$$

The solution of (81) is given by

$$
\lambda_{2}(x, y)=\frac{c(x)}{y} .
$$

Case $1.1\left(f(y)=0\right.$ and $\left.g(y)=0\left(k_{1}=k_{2}=0\right)\right)$. If we substitute (83) into (82), we obtain $c(x)=0$ and so $\lambda_{2}(x, y)=$ 0 . Therefore, $\lambda\left(x, y, y^{\prime}\right)$ is found by using (79) as follows:

$$
\lambda\left(x, y, y^{\prime}\right)=\frac{y^{\prime}}{y} .
$$

As a result, a first-order invariant $\omega\left(x, y, y^{\prime}\right)$ of $v^{[\lambda,(1)]}$ is applied to (69) then the conservation law and integration factor can be derived by using the $\lambda$-symmetry ( 84 )

$$
\omega_{y}+\frac{y^{\prime}}{y} \omega_{y^{\prime}}=0
$$

A solution of $(85)$ is

$$
\omega\left(x, y, y^{\prime}\right)=\frac{y^{\prime}}{y} .
$$

In order to express the Liénard equation (18) in terms of $\left\{x, \omega, \omega^{\prime}\right\}$, the terms $y^{\prime}$ and $y^{\prime \prime}$ can be eliminated from (86) and so the reduced form becomes

$$
\omega^{\prime}+\omega^{2}=0
$$

and the general solution of $(88)$ is

$$
\omega=\frac{1}{x-G}, \quad G \in \mathbb{R} .
$$

The integration factor corresponding to (71) can be written as

$$
\mu\left(x, y, y^{\prime}\right)=\frac{y}{y^{\prime 2}}
$$

Here, $G\left(x, \omega\left(x, y, y^{\prime}\right)\right)$ which satisfies the relation (68) is equal to conserved form

$$
D_{x}\left(x-\frac{y}{y^{\prime}}\right)=0 .
$$

Case $1.2\left(f(y)=0\right.$ and $\left.g(y)=k_{2}\left(k_{1}=0, k_{2} \neq 0\right)\right)$. By applying same process with the previous case, it is clear that in order to satisfy (82), $k_{2}$ must be equal to zero. As a result, the results in this case are similar to the Case 1.1.

Case $1.3\left(f(y)=0\right.$ and $\left.g(y)=k_{1} y\left(k_{1} \neq 0, k_{2}=0\right)\right)$. For this form for (18), similar results can be obtained with (84)-(86). Therefore the reduced form of (18) can be written as

$$
\omega^{\prime}+\omega^{2}+k_{1}=0 .
$$

The general solution of (91) is

$$
\omega=-\sqrt{a} \tan (\sqrt{a}(x-G)), \quad G \in \mathbb{R} .
$$

By using the relation (71) integration factor is given by

$$
\mu\left(x, y, y^{\prime}\right)=\frac{y}{a y^{2}+y^{\prime 2}} .
$$

In (92), $G\left(x, \omega\left(x, y, y^{\prime}\right)\right)$ corresponds to conserved form

$$
D_{x}\left(\frac{1}{\sqrt{a}}\left(x \sqrt{a}+\arctan \left(\frac{y^{\prime}}{y \sqrt{a}}\right)\right)\right)=0,
$$

which is equal to original Liénard equation (18) for this case. This relation allows us to obtain new invariant solution of (18) which is

$$
y(x)=c_{1} \cos ((K-x) \sqrt{a}),
$$

where $c_{1}$ and $K$ are constants.

Case 2. Now we analyze the case which is similar to the case in Section 3.2. As a reminder, in this section the Liénard 
equation represents linear damped systems for the choices of $f(y)=k=$ constant and $g(y)=k_{1} y+k_{2}$. For this case of $f(y)$ and $g(y)$ the third determining equation (78) becomes

$$
k_{1}-\left(k_{1} y+k_{2}\right) \lambda_{1}+k \lambda_{2}+\lambda_{2}^{2}+\lambda_{2 x}=0
$$

Now we evaluate different cases of $k_{1}$ and $k_{2}$.

Case 2.1 $\left(f(y)=k\right.$ and $\left.g(y)=0\left(k_{1}=k_{2}=0\right)\right)$. By applying similar procedure and after determination of $y^{\prime}$ and $y^{\prime \prime}$, the reduced form of Liénard equation (18) is obtained in terms of $\left\{x, \omega, \omega^{\prime}\right\}$

$$
\omega^{\prime}+\omega^{2}+k \omega=0
$$

The general solution of (97) is

$$
\omega=\frac{e^{k G} k}{e^{k G}-e^{k x}}, \quad G \in \mathbb{R} .
$$

According to (71) the integration factor is

$$
\mu\left(x, y, y^{\prime}\right)=\frac{y}{k y y^{\prime}+y^{\prime 2}}
$$

The relation related to the $G\left(x, \omega\left(x, y, y^{\prime}\right)\right)$ gives the corresponding conservation form

$$
D_{x}\left(\frac{1}{k}\left(k x-\ln \left(1+\frac{k y(x)}{y^{\prime}(x)}\right)\right)\right)=0 .
$$

Then the new invariant solution of (18) can be written as

$$
y(x)=\left(1-e^{k(C-x)}\right) c_{2}
$$

where $C$ and $c_{2}$ are constants.

Case 2.2 $\left(f(y)=k\right.$ and $\left.g(y)=k_{2}\left(k_{1}=0, k_{2} \neq 0\right)\right)$. After same manipulations it is possible to show that this case corresponds to the Case 2.1.

Case $2.3\left(f(y)=k\right.$ and $\left.g(y)=k_{1} y\left(k_{1} \neq 0, k_{2}=0\right)\right)$. According to (84)-(86), the reduced form of (18) is given by

$$
\omega^{\prime}+\omega^{2}+k \omega+k_{1}=0
$$

The general solution of (102) is

$$
\begin{array}{r}
\omega=\frac{1}{2}\left(-k+\sqrt{k^{2}-4 k_{1}} \tan \left(\frac{1}{2} \sqrt{4 k_{1}-k^{2}}(G-x)\right)\right), \\
G \in \mathbb{R} .
\end{array}
$$

By using of the relation (71) the integration factor is obtained such that

$$
\mu\left(x, y, y^{\prime}\right)=\frac{y}{a y^{2}+y^{\prime}\left(k y+y^{\prime}\right)}
$$

$G\left(x, \omega\left(x, y, y^{\prime}\right)\right)$ which is equal to conserved form can be examined from (102) and it can be written as

$$
\begin{aligned}
& D_{x}\left(\frac{1}{4 k_{1}-k^{2}}\right. \\
& \quad \times\left(4 k_{1} x-k^{2} x\right. \\
& \left.\left.\quad+2 \arctan \left(\frac{1}{4 k_{1}-k^{2}}\left(k+\frac{2 y^{\prime}}{y}\right)\right)\right)\right)=0,
\end{aligned}
$$

which satisfies the original Liénard equation (18) for this case. And so we can derive new invariant solution of (18) in the following form:

$$
y(x)=e^{1 / 2\left(-k_{1} x+2 \ln \left(\cos (1 / 2) \sqrt{4 k_{1}-k^{2}}(x-C)\right)\right)} c_{2},
$$

where $c_{2}$ and $C$ are constants.

5.1. Modified Emden Equations: $f(y)=k y ; k$ Is a Constant. It is clear that a particular solution of (31) for this special choice of $f(y)$ can be given by

$$
g(y)=\frac{1}{9} k^{2} y^{3}+k_{1} y+k_{2}
$$

In this case by the choices of the functions of $f(y)$ and $g(y)$, Liénard equation is considered in the nonlinear form [19]. We analyze four different case of $k_{1}$ and $k_{2}$. These cases are

(i) $k_{1}=k_{2}=0$,

(ii) $k_{1}=0$ and $k_{2} \neq 0$,

(iii) $k_{1} \neq 0$ and $k_{2}=0$,

(iv) $k_{1} \neq 0$ and $k_{2} \neq 0$.

In this study we consider the first and the third cases since one can find only lambda function for the cases in which it is assumed in the linear form.

5.1.1. Modified Emden Equation $\left(k_{1}=k_{2}=0\right)$. Corresponding Liénard equation for $k_{1}=k_{2}=0$ is

$$
y^{\prime \prime}+k y^{\prime} y+\left(\frac{1}{9}\right) k^{2} y^{3}=0
$$

which is nonlinear ordinary differential equation. In order to obtain $\lambda$-symmetries the determining equations (76)-(78) should be evaluated together. So the results of these equations give $\lambda$-symmetry (79) such that

$$
\lambda\left(x, y, y^{\prime}\right)=\frac{y^{\prime}}{y}+\frac{k y}{3},
$$

and by substituting (109) into (69) we obtain

$$
\omega_{y}+\left(\frac{y^{\prime}}{y}+\frac{k y}{3}\right) \omega_{y^{\prime}}=0 .
$$


It is clear that a solution of this (110) is

$$
\omega\left(x, y, y^{\prime}\right)=\frac{k y^{2}+3 y^{\prime}}{3 y} .
$$

By using (111) $y^{\prime}$ and $y^{\prime \prime}$ can be derived in terms of $w$ and so Liénard equation can be written as

$$
\omega^{\prime}+\omega^{2}=0
$$

which is the reduced form. The general solution of (112) is

$$
\omega=\frac{1}{x-G}, \quad G \in \mathbb{R}
$$

Integration factor corresponding to (71) is

$$
\mu\left(x, y, y^{\prime}\right)=\frac{9 y}{\left(k y^{2}+3 y^{\prime}\right)^{2}} \text {. }
$$

It is clear that $G\left(x, \omega\left(x, y, y^{\prime}\right)\right)$ is equivalent to conserved form

$$
D_{x}\left(\frac{-3 y+k y^{2} x+3 y^{\prime} x}{k y^{2}+3 y^{\prime}}\right)=0 \text {, }
$$

and the reduced equation of (108) can be derived as

$$
\frac{-3 y(x)+k y(x)^{2} x+3 y^{\prime}(x) x}{k y(x)^{2}+3 y(x)^{\prime}}-C=0 .
$$

The solution of (116) gives the new invariant solution of (108)

$$
y(x)=\frac{6(C-x)}{2 C k x-k x^{2}-6 c_{1}},
$$

where $C$ and $c_{1}$ are constants.

5.1.2. Modified Emden Equation with Linear Term $\left(k_{1} \neq 0\right.$ and $\left.k_{2}=0\right)$. Choosing $k_{1}$ as arbitrary $k_{2}=0$ Liénard equation is obtained as a nonlinear ordinary differential equation in the form

$$
y^{\prime \prime}+k y y^{\prime} \frac{1}{9} k^{2} y^{3}+k_{1} y
$$

By the same way and for this case the reduced form of (18) can be obtained

$$
\omega^{\prime}+\omega^{2}+k_{1}=0
$$

The general solution of (119) is

$$
\omega=-\sqrt{k_{1}} \tan \left(\sqrt{k_{1}}(x-G)\right), \quad G \in \mathbb{R} .
$$

According to (71) the integration factor is examined

$$
\mu\left(x, y, y^{\prime}\right)=\frac{9 y}{\left(k y^{2}+3 y^{\prime}\right)^{2}+9 k_{1} y^{2}} .
$$

It is clear that $G\left(x, \omega\left(x, y, y^{\prime}\right)\right)$ is equivalent to conserved form

$$
D_{x}\left(\left(x \arctan \left(\frac{k y^{2}+3 y^{\prime}}{3 y \sqrt{k_{1}}}\right)\right)\right)=0 .
$$

The reduced equation of (118) can be derived as

$$
x \arctan \left(\frac{k y^{2}+3 y^{\prime}}{3 y \sqrt{k_{1}}}\right)-C=0,
$$

and the solution of (123) gives the new invariant solution of (108)

$$
y(x)=\frac{3 \sqrt{k_{1}} \cos \left((C-x) \sqrt{k_{1}}\right)}{3 \sqrt{k_{1}} c_{1}-k \sin \left((C-x) \sqrt{k_{1}}\right)},
$$

where $C$ and $c_{1}$ are constants.

5.2. Alternative Approach for the Consideration of $f(y)$ and $g(y)$. Alternatively, to get a classification, we first define $f(y)$ function and then try to determine $\lambda_{1}, \lambda_{2}$, and $g(y)$ functions. The corresponding subcases are given below.

5.3. $f(y)=a y^{n}+b y^{n-1}+c,(n \neq-2, n \neq-1)$. where $a, b$, and $c$ are arbitrary constants. For this case of $f(y)$ we get (80) of the form

$$
\frac{2 \lambda_{2}}{y}+\lambda_{2 y}+a n y^{n-1}+b(n-1) y^{n-2}=0 .
$$

A particular solution of this equation gives the $\lambda_{2}(x, y)$ such that

$$
\lambda_{2}(x, y)=-y^{n-2}\left(\frac{b y(n-1)}{n+1}+\frac{a n y^{2}}{n+2}\right) .
$$

Hence, $\lambda$-symmetry can be written by using (79)

$$
\lambda\left(x, y, y^{\prime}\right)=\frac{y^{\prime}}{y}-y^{n-2}\left(\frac{b y(n-1)}{n+1}+\frac{a n y^{2}}{n+2}\right) .
$$

If we substitute $\lambda_{1}$ and $\lambda_{2}$ into (78), then the new form of differential equation is obtained in terms of unknown function $g(y)$

$$
\begin{aligned}
& g^{\prime}(y)-\frac{g(y)}{y}+y^{n-2}\left(\frac{b y(n-1)}{n+1}+\frac{a n y^{2}}{n+2}\right) \\
& \quad \times\left(y^{n-2}\left(\frac{b y(n-1)}{n+1}+\frac{a n y^{2}}{n+2}\right)-a y^{n}-b y^{n-1}-c\right)=0,
\end{aligned}
$$

then the general solution of (128) is given by

$$
\begin{aligned}
g(y)= & y c_{1}+\frac{1}{\left(n^{2}+3 n+2\right)^{2}} \\
& \times\left(y^{n-1}(b(n+2)+a(n+1) y)\right. \\
& \times\left(c y\left(n^{2}+3 n+2\right)\right. \\
& \left.\left.\quad+y^{n}(b(n+2)+a(n+1) y)\right)\right),
\end{aligned}
$$


where $c_{1}$ is constant. Thus the Liénard equation for these special cases for $f(y)$ and $g(y)(18)$ becomes

$$
\begin{aligned}
y^{\prime \prime}+ & y^{\prime}\left(a y^{n}+b y^{n-1}+c\right)+y c_{1}+\frac{1}{\left(n^{2}+3 n+2\right)^{2}} \\
& \times\left(y^{n-1}(b(n+2)+a(n+1) y)\right. \\
& \times\left(c y\left(n^{2}+3 n+2\right)\right. \\
& \left.\left.+y^{n}(b(n+2)+a(n+1) y)\right)\right)=0 .
\end{aligned}
$$

In order to obtain the conservation law and the integration factor by using $\lambda$-symmetry (127), a first-order invariant $\omega\left(x, y, y^{\prime}\right)$ of $v^{[\lambda,(1)]}$ is obtained in the following form from the relation (69):

$$
\omega_{y}+\left(\frac{y^{\prime}}{y}-y^{n-2}\left(\frac{b y(n-1)}{n+1}+\frac{a n y^{2}}{n+2}\right)\right) \omega_{y^{\prime}}=0
$$

which has a solution of the form

$$
\begin{aligned}
& \omega\left(x, y, y^{\prime}\right) \\
& =\frac{1}{\left(n^{2}+3 n+2\right)} \\
& \quad \times\left(2 b y^{n}+b n y^{n}+a y^{n+1}+a n y^{n+1}+2 y^{\prime}+3 n y^{\prime}+n^{2} y^{\prime}\right) .
\end{aligned}
$$

So in terms of $\left\{x, \omega, \omega^{\prime}\right\}$ the Liénard equation (18) can be written as

$$
\omega^{\prime}+\omega^{2}+b \omega+c_{1}=0
$$

And the general solution of (133) is given by

$$
\omega=\frac{1}{2}\left(-b \sqrt{4 c_{1}-b^{2}} \tan \left(\frac{1}{2} \sqrt{4 c_{1}-b^{2}}(G-x)\right)\right), \quad G \in \mathbb{R} .
$$

According to (71) we obtain the integration factor as

$$
\begin{gathered}
\mu\left(x, y, y^{\prime}\right)=\left(y^{2}\left(n^{2}+3 n+2\right)^{2}\right) \\
\times\left(\left(c\left(n^{2}+3 n+2\right) y\right.\right. \\
+2\left(b y^{n}(n+2)+(n+1)\right. \\
\left.\left.\times\left(a y^{n+1}+(n+2) y^{\prime}\right)\right)\right)^{2} \\
\left.-y^{2}\left(n^{2}+3 n+2\right)^{2}\right)^{-1} .
\end{gathered}
$$

It is the fact that $G\left(x, \omega\left(x, y, y^{\prime}\right)\right)$ is equivalent to the following conserved form

$$
\begin{aligned}
& D_{x}\left(\frac{1}{b^{2}-4 c_{1}}\right. \\
& \times\left(b^{2}-4 c_{1} x-2 \sqrt{4 c_{1}-b^{2}}\right. \\
& \times \arctan \left(\frac{c}{\sqrt{4 c_{1}-b^{2}}}\right. \\
& +2\left(b y^{n}(n+2)+(n+1)\right. \\
& \left.\times\left(a y^{n+1}+(n+2) y^{\prime}\right)\right) \\
& \times\left(\sqrt{4 c_{1}-b^{2}}\right. \\
& \left.\left.\left.\times\left(n^{2}+3 n+2\right) y\right)^{-1}\right)\right)=0
\end{aligned}
$$

which gives the original Liénard equation given in the form (130). It is possible to say that the solution of reduced equation of (130) cannot be solved for the general $n$. But, if we choose specifically $n=1$ then the reduced form of (130) is

$$
\begin{aligned}
& \frac{1}{b^{2}-4 c_{1}} \\
& \times\left(b^{2}-4 c_{1} x-2 \sqrt{4 c_{1}-b^{2}}\right. \\
& \left.\quad \times \arctan \left(\frac{3 b y(x)+6 y(x)^{\prime}+2 a y(x)^{2}}{3 y(x) \sqrt{4 c_{1}-b^{2}}}\right)\right)
\end{aligned}
$$

$$
-k_{1}=0
$$

where $k_{1}$ is a constant. It is a fact that this type of Liénard equation (130) for the case $n=1$ cannot be solved by known methods in the literature. But in this analysis, the solution of reduced equation (127) as a new invariant solution of (130) can be obtained as

$$
\begin{aligned}
y(x)= & \left(6 c_{1} \cos \left(\frac{1}{2} \sqrt{4 c_{1}-b^{2}}\left(x-k_{1}\right)\right)\right) \\
& \times\left(6 k_{2} e^{b x / 2}-a b \cos \left(\frac{1}{2} \sqrt{4 c_{1}-b^{2}}\left(k_{1}-x\right)\right)\right. \\
& \left.-a \sqrt{4 c_{1}-b^{2}} \sin \left(\frac{1}{2} \sqrt{4 c_{1}-b^{2}}\left(k_{1}-x\right)\right)\right)^{-1}
\end{aligned}
$$

where $k_{2}$ is a constant. 
In this case, it is possible to check that two specific cases for $n$ should be analyzed separately such that $(n=-2, n=$ -1 ). We investigate these, respectively, in the following subcases.

5.3.1. $f(y)=\left(a / y^{2}\right)+\left(b / y^{3}\right)+c, n=-2$. By using this form of $f(y)$, (80) becomes

$$
\frac{2 \lambda_{2}}{y}+\lambda_{2 y}-\frac{3 b}{y^{4}}-\frac{2 a}{y^{3}}=0 .
$$

A particular solution of $\lambda_{2}(x, y)$ from differential equation (139) is

$$
\lambda_{2}(x, y)=\frac{2 a y \ln y-3 b}{y^{3}}
$$

Thus the $\lambda$-symmetry is obtained from the relation (79) as

$$
\lambda\left(x, y, y^{\prime}\right)=\frac{y^{\prime}}{y}+\frac{2 a y \ln y-3 b}{y^{3}} .
$$

The differential equation (78) can be rewritten in terms of unknown function $g(y)$ by considering $\lambda_{1}$ and $\lambda_{2}$ in the following form:

$$
\begin{aligned}
g^{\prime}(y) & -\frac{g(y)}{y}+\frac{1}{y^{4}}\left(2 a \ln y-\frac{3 b}{y}\right)^{2} \\
& +\frac{1}{y^{2}}\left(\left(\frac{a}{y^{2}}+\frac{b}{y^{3}}+c\right)\left(2 a \ln y-\frac{3 b}{y}\right)\right)=0
\end{aligned}
$$

hence, the general solution of (142) is found as follows:

$$
\begin{aligned}
& g(y)=y c_{1}+\frac{1}{4 y^{5}} \\
& \times((-2 b+a y+2 a y \ln y) \\
& \left.\times\left(-2 b+a y+2 c y^{3}+2 a y \ln y\right)\right),
\end{aligned}
$$

where $c_{1}$ is constant. So we can rewrite the Liénard equation (18) for this case of $f(y)$ and $g(y)$

$$
\begin{aligned}
y^{\prime \prime}+y^{\prime}\left(\frac{a}{y^{2}}+\frac{b}{y^{3}}+c\right)+y c_{1} \\
+\frac{1}{4 y^{5}}((-2 b+a y+2 a y \ln y) \\
\left.\quad \times\left(-2 b+a y+2 c y^{3}+2 a y \ln y\right)\right) .
\end{aligned}
$$

If we substitute $\lambda$-symmetry into (69), the relation

$$
\omega_{y}+\left(\frac{y^{\prime}}{y}+\frac{2 a y \ln y-3 b}{y^{3}}\right) \omega_{y^{\prime}}=0
$$

is obtained. A solution of this equation is

$$
\omega\left(x, y, y^{\prime}\right)=\frac{-2 b+a y+2 y^{2} y^{\prime}+2 a y \ln y}{2 y^{3}} .
$$

In order to obtain Liénard equation (18) in terms of $\left\{x, \omega, \omega^{\prime}\right\}$, the equalities $y^{\prime}$ and $y^{\prime \prime}$ can be simplified (146). And so the reduced form is

$$
\omega^{\prime}+\omega^{2}+b \omega+c_{1}=0
$$

and the general solution of (147) is given by

$$
\omega=\frac{1}{2}\left(-b \sqrt{4 c_{1}-b^{2}} \tan \left(\frac{1}{2} \sqrt{4 c_{1}-b^{2}}(G-x)\right)\right), \quad G \in \mathbb{R}
$$

thus the integration factor corresponding to (71) is

$$
\begin{aligned}
\mu\left(x, y, y^{\prime}\right)= & \left(y^{6}\right) \\
\times & \left(\left(-2 b+y\left(a+c y^{2}+2 y y^{\prime}\right)+2 a y \ln y\right)^{2}\right. \\
& \left.\quad-\left(c^{2}-4 c_{1}\right) y^{6}\right)^{-1} .
\end{aligned}
$$

It is clear that $G\left(x, \omega\left(x, y, y^{\prime}\right)\right)$ is equal to the conserved form

$$
\begin{aligned}
& D_{x}\left(\frac{1}{b^{2}-4 c_{1}}\right. \\
& \left.\quad \times\left(\frac{c^{2}-4 c_{1} x-2 \sqrt{4 c_{1}-b^{2}}}{\quad} \quad\left(\frac{c y^{3}-2 b+a y+2 y^{2} y^{\prime}+2 a y \ln y}{y^{3} \sqrt{4 c_{1}-b^{2}}}\right)\right)\right) \\
& =0, \quad
\end{aligned}
$$

which gives the original Liénard equation such as the form (143).

5.3.2. $f(y)=(a / y)+\left(b / y^{2}\right)+c, n=-1$. Equation (80) is found in the following form:

$$
\frac{2 \lambda_{2}}{y}+\lambda_{2 y}-\frac{2 b}{y^{3}}-\frac{a}{y^{2}}=0 .
$$

A particular solution of (151) is equal to

$$
\lambda_{2}(x, y)=\frac{a y+2 b \ln y}{y^{2}} \text {. }
$$

By substituting (79) and (152) into (74) we have

$$
\lambda\left(x, y, y^{\prime}\right)=\frac{y^{\prime}}{y}+\frac{a y+2 b \ln y}{y^{2}} .
$$


If $\lambda_{1}$ and $\lambda_{2}$ are inserted to (78) the differential equation is obtained in terms of $g(y)$

$$
\begin{aligned}
& g^{\prime}(y)-\frac{g(y)}{y}+\frac{(a y+2 b \ln y)^{2}}{y^{4}} \\
& +\frac{1}{y^{2}}\left(\frac{a}{y}+\frac{b}{y^{2}}+c\right)(a y+2 b \ln y)=0
\end{aligned}
$$

the general solution of (154) is

$$
\begin{aligned}
g(y)= & y c_{1}+\frac{1}{4 y^{3}} \\
& \times\left((b+2 a y+2 b \ln y)\left(2 b \ln y+b+2 c y^{2}+2 a y\right)\right),
\end{aligned}
$$

where $c_{1}$ is constant. For this case of $f(y)$ and $g(y)$ Liénard equation (18) corresponds to

$$
\begin{aligned}
y^{\prime \prime} & +y^{\prime}\left(\frac{a}{y}+\frac{b}{y^{2}}+c\right)+y c_{1}+\frac{1}{4 y^{3}} \\
& \times\left((b+2 a y+2 b \ln y)\left(2 b \ln y+b+2 c y^{2}+2 a y\right)\right) .
\end{aligned}
$$

According to (69) we obtain

$$
\omega_{y}+\left(\frac{y^{\prime}}{y}+\frac{a y+2 b \ln y}{y^{2}}\right) \omega_{y^{\prime}}=0
$$

A solution of (157) is

$$
\omega\left(x, y, y^{\prime}\right)=\frac{b+2 a y+2 y y^{\prime}+2 b \ln y}{2 y^{2}} .
$$

By using (158) the reduced form of Liénard equation (18) in terms of $\left\{x, \omega, \omega^{\prime}\right\}$ can be written in the form

$$
\omega^{\prime}+\omega^{2}+c \omega+c_{1}=0
$$

and the general solution of (159) is

$$
\omega=\frac{1}{2}\left(-b \sqrt{4 c_{1}-c^{2}} \tan \left(\frac{1}{2} \sqrt{4 c_{1}-c^{2}}(G-x)\right)\right), \quad G \in \mathbb{R} .
$$

According to (71) the integration factor is equal to

$$
\begin{aligned}
\mu\left(x, y, y^{\prime}\right)= & \left(4 y^{3}\right) \\
& \quad \times\left(4 y^{4} c_{1}+\left(b+2 y\left(a+y^{\prime}\right)+2 b \ln y\right)\right. \\
& \left.\quad \times\left(b+2 y\left(a+c y+y^{\prime}\right)+2 b \ln y\right)\right)^{-1}
\end{aligned}
$$

$G\left(x, \omega\left(x, y, y^{\prime}\right)\right)$ that is reduced to (160) corresponds to conserved form of (18)

$$
\begin{aligned}
& D_{x}\left(\frac{1}{c^{2}-4 c_{1}}\right. \\
& \left.\quad \times\left(\frac{c^{2}-4 c_{1} x-2 \sqrt{4 c_{1}-c^{2}}}{\quad} \quad\left(\frac{c+2 b+4 a y+4 y y^{\prime}+4 b \ln y}{\sqrt{4 c_{1}-c^{2}}}\right)\right)\right)=0,
\end{aligned}
$$

which is equal to the original Liénard equation of the form (156).

5.4. $f(y)=a \cosh y+b$. According to (80) the differential equation in terms of $\lambda_{2}$ is given by

$$
\frac{2 \lambda_{2}}{y}+\lambda_{2 y}+a \sinh y=0 \text {. }
$$

The solution of above equation is found as follows:

$$
\lambda_{2}(x, y)=\frac{a\left(2 y \sinh y-\left(2+y^{2}\right) \cosh y\right)}{y^{2}} .
$$

The $\lambda$-symmetry considered as in the linear form of functions of $\lambda_{1}$ and $\lambda_{2}$ is equal to

$$
\lambda\left(x, y, y^{\prime}\right)=\frac{y^{\prime}}{y}+\frac{a\left(2 y \sinh y-\left(2+y^{2}\right) \cosh y\right)}{y^{2}} .
$$

Thus, (78) that includes the $g(y)$ function is obtained as the form

$$
\begin{aligned}
g^{\prime}(y) & -\frac{g(y)}{y} \\
- & \frac{a(a \cosh y+b)\left(\left(2+y^{2}\right) \cosh y-2 y \sinh y\right)}{y^{2}} \\
+ & \frac{\left(\left(2+y^{2}\right) \cosh y-2 y \sinh y\right)^{2} a^{2}}{y^{4}}=0 .
\end{aligned}
$$

The general solution of (166) is

$$
\begin{aligned}
g(y)= & y c_{1}+\frac{1}{y^{3}} \\
& \times(a(y \sinh y-\cosh y) \\
& \quad \times(y(a \sinh y+b y)-a \cosh y)),
\end{aligned}
$$


where $c_{1}$ is a constant. Consequently, Liénard equation (18) for this case of $f(y)$ and $g(y)$ is found as below

$$
\begin{aligned}
& y^{\prime \prime}+y^{\prime}(a \cosh y+b)+y c_{1} \\
& +\frac{1}{y^{3}}(a(y \sinh y-\cosh y) \\
& \quad \times(y(a \sinh y+b y)-a \cosh y)) .
\end{aligned}
$$

By consideration of the relation (69) the equation

$$
\omega_{y}+\left(\frac{y^{\prime}}{y}+\frac{a\left(2 y \sinh y-\left(2+y^{2}\right) \cosh y\right)}{y^{2}}\right) \omega_{y^{\prime}}=0
$$

is derived. A solution of (169) is equal to

$$
\omega\left(x, y, y^{\prime}\right)=\frac{y^{\prime} y-a \cosh y+a y \sinh y}{y^{2}} .
$$

In order to find the reduced form of Liénard equation (18), $y^{\prime}$ and $y^{\prime \prime}$ can be examined (170). Therefore we can write the following equation:

$$
\omega^{\prime}+\omega^{2}+c \omega+c_{1}=0,
$$

and the general solution of (171) is equal to

$$
\omega=\frac{1}{2}\left(-c \sqrt{4 c_{1}-c^{2}} \tan \left(\frac{1}{2} \sqrt{4 c_{1}-c^{2}}(G-x)\right)\right), \quad G \in \mathbb{R} .
$$

The integration factor corresponding to (71) is

$$
\begin{aligned}
& \mu\left(x, y, y^{\prime}\right) \\
& =\left(y^{3}\right) \\
& \quad \times\left(c_{1} y^{4}+\left(-a \cosh y+y\left(y^{\prime}+a \sinh y\right)\right)\right. \\
& \left.\quad \times\left(-a \cosh y+y\left(b y+y^{\prime}+-a \cosh y\right)\right)\right)^{-1} .
\end{aligned}
$$

The conserved form $G\left(x, \omega\left(x, y, y^{\prime}\right)\right)$ can be derived from (172)

$$
\begin{aligned}
& D_{x}\left(\frac{1}{b^{2}-4 c_{1}}\right. \\
& \times\left(b^{2}-4 c_{1} x-2 \sqrt{4 c_{1}-b^{2}}\right. \\
& \times \arctan \left(\frac{1}{\sqrt{4 c_{1}-b^{2}}}\right. \\
& \quad \times(b+2 \\
& \left.\left.\left.\left.\quad \times\left(\frac{y^{\prime} y-a \cosh y+a y \sinh y}{y^{2}}\right)\right)\right)\right)\right)
\end{aligned}
$$$$
=0 \text {, }
$$

which gives the original Liénard equation of the form (168).

5.5. $f(y)=a e^{b y}$. For this case of $f(y)$ the differential equation in terms of $\lambda_{2}$ is written as follows:

$$
\frac{2 \lambda_{2}}{y}+\lambda_{2 y}+a b e^{b y}=0 .
$$

A particular solution of (175) is

$$
\lambda_{2}(x, y)=-\frac{a e^{b y}\left(2-2 b y+b^{2} y^{2}\right)}{b^{2} y^{2}} .
$$

By substituting (79) and (176) into (74) we obtain

$$
\lambda\left(x, y, y^{\prime}\right)=\frac{y^{\prime}}{y}-\frac{a e^{b y}\left(2-2 b y+b^{2} y^{2}\right)}{b^{2} y^{2}} .
$$

By applying $\lambda_{1}$ and $\lambda_{2}$ to (78) the differential equation which depends on unknown function $g(y)$ is derived

$$
\begin{gathered}
g^{\prime}(y)-\frac{g(y)}{y}-\frac{a^{2} e^{2 b y}\left(2-2 b y+b^{2} y^{2}\right)}{b^{2} y^{2}} \\
+\frac{a^{2} e^{2 b y}\left(2-2 b y+b^{2} y^{2}\right)}{b^{4} y^{4}}=0,
\end{gathered}
$$

and the general solution of (178) is equal to

$$
g(y)=y c_{1}+\frac{a^{2} e^{2 b y}(b y-1)^{2}}{b^{4} y^{3}},
$$


where $c_{1}$ is constant. The Liénard equation (18) for this case of $f(y)$ and $g(y)$ corresponds to the following form:

$$
y^{\prime \prime}+y^{\prime} a e^{b y}+y c_{1}+\frac{a^{2} e^{2 b y}(b y-1)^{2}}{b^{4} y^{3}}=0 .
$$

Equation (69) can be written as

$$
\omega_{y}+\left(\frac{y^{\prime}}{y}-\frac{a e^{b y}\left(2-2 b y+b^{2} y^{2}\right)}{b^{2} y^{2}}\right) \omega_{y^{\prime}}=0
$$

A solution of this equation is found as below

$$
\omega\left(x, y, y^{\prime}\right)=\frac{-a e^{b y}+a b e^{b y} y+b^{2} y y^{\prime}}{b^{2} y^{2}} .
$$

The reduced form of Liénard equation (18) in terms of variables $\left\{x, \omega, \omega^{\prime}\right\}$ becomes

$$
\omega^{\prime}+\omega^{2}+c_{1}=0
$$

the general solution of (183) is given by

$$
\omega=-\sqrt{c_{1}} \tan \left(\sqrt{c_{1}}(x-G)\right), \quad G \in \mathbb{R} .
$$

As a result, the integration factor is obtained in the form by using (71)

$$
\mu\left(x, y, y^{\prime}\right)=\frac{b^{4} y^{3}}{c_{1} b^{4} y^{4}+\left(a e^{b y}(b y-1)+b^{2} y y^{\prime}\right)^{2}} .
$$

The conserved form associated with $G\left(x, \omega\left(x, y, y^{\prime}\right)\right)$ is

$$
D_{x}\left(\frac{1}{\sqrt{c_{1}}}\left(x \sqrt{c_{1}} \arctan \left(\frac{-a e^{b y}+a b e^{b y} y+b^{2} y y^{\prime}}{b^{2} \sqrt{c_{1}} y^{2}}\right)\right)\right)=0,
$$

which gives the original Liénard equation such as the form (180).

\section{Conclusion}

In this study we apply partial Noether symmetry and $\lambda$ symmetry approaches to Liénard equation, which has an important role in terms of physics and mechanics. Based on the studies of partial Noether operators, we derive partial Lagrangian of Liénard equation, which leads to obtain partial Noether symmetries. Since the Liénard equation includes two arbitrary functions such as $f(y)$ and $g(y)$, we evaluate different cases of these functions for the classification. Partial Noether symmetries are investigated for two different problems related to the linear damped and undamped systems. And we derive new first integrals (conserved forms) corresponding to each partial Noether symmetry.

Alternatively, in the literature the $\lambda$-symmetry concept is used to study integration factors, first integrals, and invariant solutions of ordinary differential equations. Hence, the other part of this study is based on the relation between integrating factors and $\lambda$-symmetries for Liénard equation related to some mechanics problems. In fact, in this approach the determining equation for the differential equation includes not only infinitesimal functions but also $\lambda$ function, which are unknown functions but it is not possible to get a solution of determining equations easily. In order to overcome this difficulty for determining equations, the $\lambda$ function is assumed to be in a linear form. In this way the system of determining equations which include partial differential equations can be solved easily. In the first part of the study we consider same functions $f(y)$ and $g(y)$ considered in the case of partial Noether symmetries. Based on these functions we study $\lambda$ symmetries and first integrals. Then we conclude that it is possible to obtain new first integral by using $\lambda$-symmetry approach, which is different from partial Noether symmetry approach.

Furthermore, we analyze only some specific cases of arbitrary function $f(y)$ to determine $\lambda$-symmetries. As a result of determining equations we find different $g(y)$ functions corresponding to each of $f(y)$ functions and obtain new $\lambda$-symmetries. By using $\lambda$-symmetries we obtain new reduced forms, integration factors, and conserved forms of highly nonlinear Liénard type equation. We present that the conserved forms lead to new invariant solutions of corresponding classical mechanics problems, which are considered by different approaches in the literature.

\section{Conflict of Interests}

The authors declare that there is no conflict of interests regarding the publication of this paper.

\section{References}

[1] E. Noether, "Invariante Variationsprobleme," Nachrichten von der Gesellschaft der Wissenschaften zu Göttingen, MathematischPhysikalische Klasse, vol. 2, pp. 235-257, 1918, English Translation in Transport Theory and Statistical Physics, vol. 1, no. 3, pp. 186-207, 1971.

[2] G. W. Bluman and S. Kumei, Symmetries and Differential Equations, vol. 81 of Applied Mathematical Sciences, Springer, New York, NY, USA, 1989.

[3] L. V. Ovsjannikov, Group Analysis of Differential Equations, Nauka, Moscow, Russia, 1978.

[4] N. H. Ibragimov, Ed., CRC Handbook of Lie Group Analysis of Differential Equations, vol. 1, CRC Press, New York, NY, USA, 1993.

[5] P. J. Olver, Applications of Lie Groups to Differential Equations, Springer, New York, NY, USA, 1986.

[6] H. Stephani, Differential Equations: Their Solutions Using Symmetries, Cambridge University Press, Cambridge, UK, 1989.

[7] E. Yaşar and T. Özer, "On symmetries, conservation laws and invariant solutions of the foam-drainage equation," International Journal of Non-Linear Mechanics, vol. 46, no. 2, pp. 357362,2011

[8] T. Özer, N. Antar, and D. Sahin, "Lie group analysis of gravity currents," Nonlinear Analysis: Real World Applications, vol. 11, no. 2, pp. 978-994, 2010.

[9] T. Özer and E. Yaşar, "Conservation laws for one-layer shallow water wave systems," Nonlinear Analysis: Real World Applications, vol. 11, no. 2, pp. 838-848, 2010. 
[10] T. Özer, "Symmetry group classification for one-dimensional elastodynamics problems in nonlocal elasticity," Mechanics Research Communications, vol. 30, no. 6, pp. 539-546, 2003.

[11] T. Ozer, "On symmetry group properties and general similarity forms of the Benney equations in the Lagrangian variables," Journal of Computational and Applied Mathematics, vol. 169, no. 2, pp. 297-313, 2004.

[12] T. Özer, "Symmetry group classification for two-dimensional elastodynamics problems in nonlocal elasticity," International Journal of Engineering Science, vol. 41, no. 18, pp. 2193-2211, 2003.

[13] S. N. Pandey, P. S. Bindu, M. Senthilvelan, and M. Lakshmanan, "A group theoretical identification of integrable cases of the Liénard-type equation $\ddot{x}+f(x) \dot{x}+g(x)=0$. I. Equations having nonmaximal number of Lie point symmetries," Journal of Mathematical Physics, vol. 50, no. 8, Article ID 082702, 19 pages, 2009.

[14] A. H. Kara, F. M. Mahomed, I. Naeem, and C. Wafo Soh, "Partial Noether operators and first integrals via partial Lagrangians," Mathematical Methods in the Applied Sciences, vol. 30, no. 16, pp. 2079-2089, 2007.

[15] C. Muriel and J. L. Romero, "First integrals, integrating factors and $\lambda$-symmetries of second-order differential equations," Journal of Physics A: Mathematical and Theoretical, vol. 42, no. 36, Article ID 365207, 17 pages, 2009.

[16] C. Muriel and J. L. Romero, "New methods of reduction for ordinary differential equations," IMA Journal of Applied Mathematics, vol. 66, no. 2, pp. 111-125, 2001.

[17] C. Muriel and J. L. Romero, "Integrating factors and $\lambda$ symmetries," Journal of Nonlinear Mathematical Physics, vol. 15, no. 3, pp. 300-309, 2008.

[18] C. Muriel and J. L. Romero, " $C^{\infty}$-symmetries and reduction of equations without lie point symmetries," Journal of Nonlinear Mathematical Physics, vol. 13, no. 1, pp. 167-188, 2003.

[19] E. Yaşar, "Integrating factors and first integrals for Liénard type and frequency-damped oscillators," Mathematical Problems in Engineering, vol. 2011, Article ID 916437, 10 pages, 2011. 


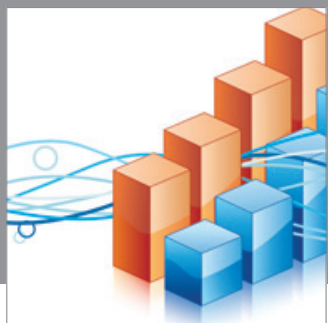

Advances in

Operations Research

mansans

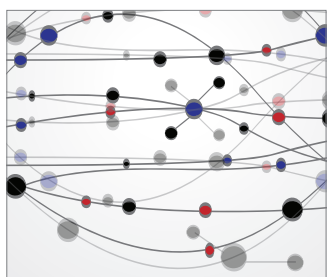

The Scientific World Journal
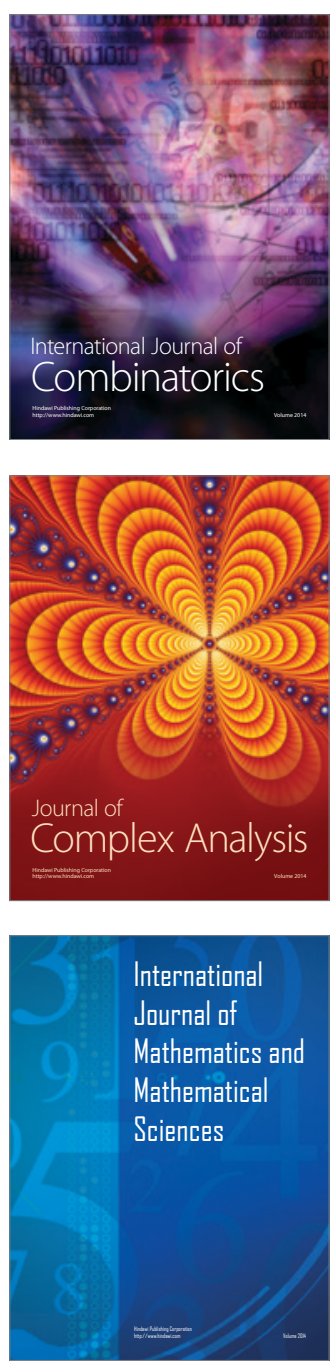
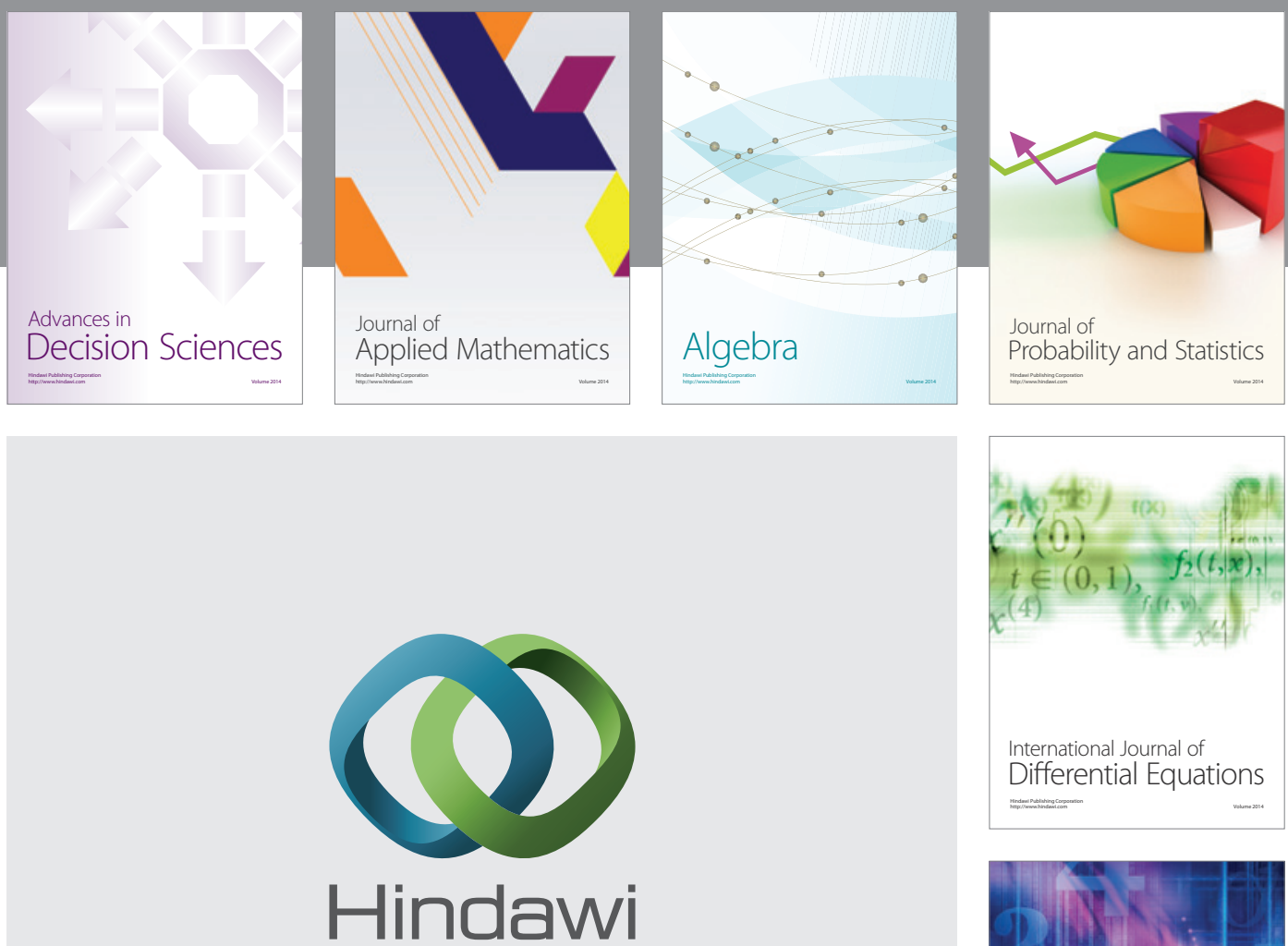

Submit your manuscripts at http://www.hindawi.com
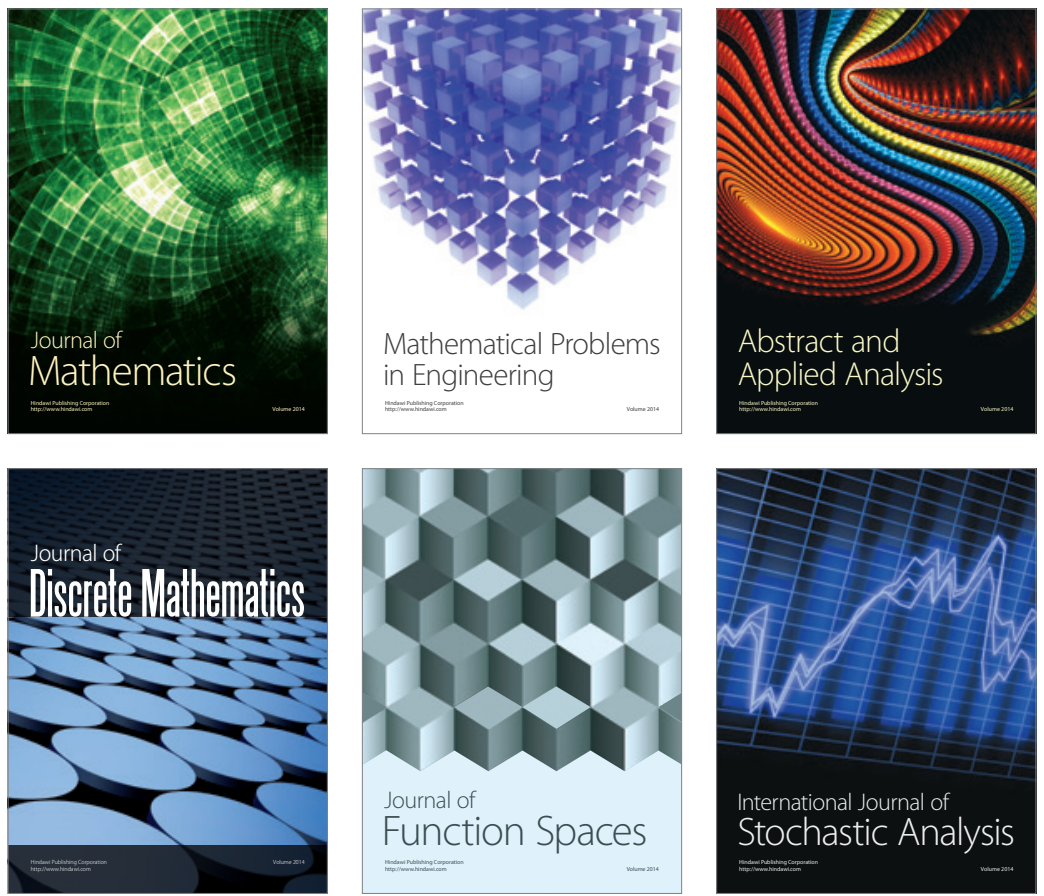

Journal of

Function Spaces

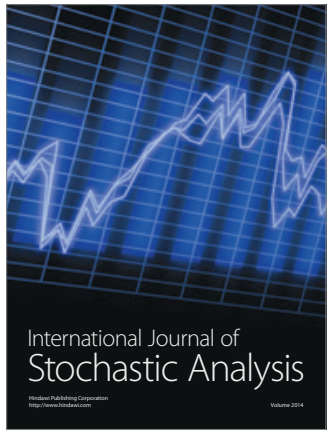

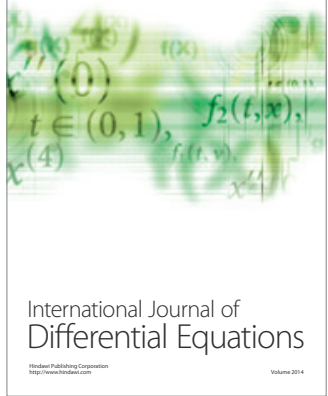
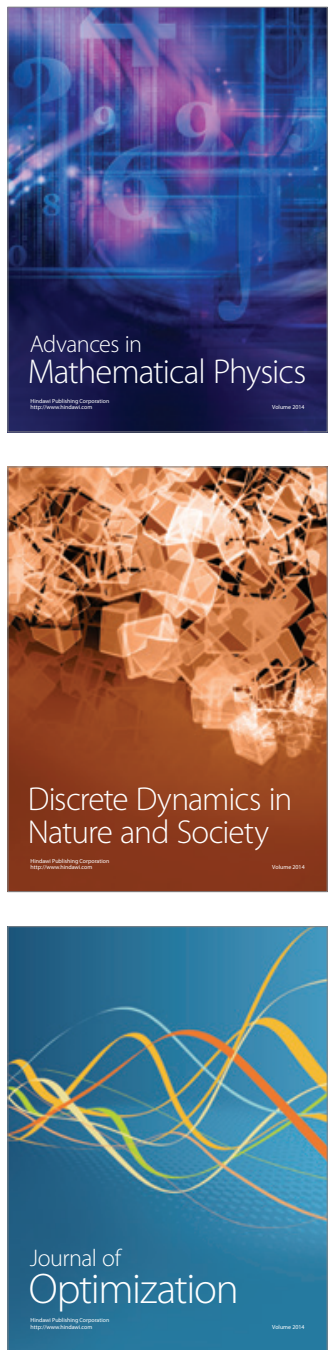